\title{
Lifetime Optimization via Network Sectoring in Cooperative Wireless Sensor Networks
}

\author{
Hadi Jamali Rad ${ }^{1}$, Bahman Abolhassani ${ }^{1}$, Mohammad Abdizadeh ${ }^{2}$ \\ ${ }^{1}$ School of Electrical Engineering, Iran University of Science and Technology, Tehran, Iran \\ ${ }^{2}$ School of Electrical Engineering, Sharif University of Technology, Tehran, Iran \\ E-mail:h.jamali@ee.iust.ac.ir,abolhassani@iust.ac.ir,abdizadeh@ee.sharif.ir \\ Received October 3, 2010; revised October 12, 2010; accepted October 14, 2010
}

\begin{abstract}
Employing cooperative communication in multihop wireless sensor networks provides the network with significant energy efficiency. However, the lifetime of such a network is directly dependant upon the lifetime of each of its individual sections (or clusters). Ignoring the fact that those sections close to sink have to forward more data (their own data plus the data received from the previous sections) and hence die sooner with considering equal section sizes, leads to a sub-optimal lifetime. In this paper, we optimize the section sizes of a multihop cooperative WSN so that it maximizes the network lifetime. Simulation results demonstrate a significant lifetime enhancement for the proposed optimal sectoring.
\end{abstract}

Keywords: Wireless Sensor Network (WSN), Cooperative Communications, Virtual Multi-Input-Multi-Output (V-MIMO), Energy Efficiency

\section{Introduction}

Wireless sensor networks (WSNs) consist of a large number of tiny sensor nodes deployed over a large area. Such wireless nodes typically operate with small batteries for which replacement, when possible is very difficult and expensive. Thus, in many practical scenarios, the wireless nodes should operate for many years without any battery replacement or recharge. Hence, minimizing the power consumption in different functional layers of wireless nodes is a very challenging design consideration. Furthermore, affected by channel impairments, energy constraints of the sensor nodes are the main limiting factors of the system performance of WSNs and therefore power efficiency is of special importance in such networks.

Diversity techniques have been proposed to provide a wireless network with reliable and power efficient communications [1,2]. However, tiny sensor nodes cannot accommodate multiple antennas and hence the implementation of MIMO-based communications in a wireless sensor network (WSN) requires cooperation among sensor nodes [3,4]. These sensor nodes can be considered as a virtual MIMO (V-MIMO) unit.

Moreover, in large WSNs, multihop communications are used instead of long-haul transmission to reduce high energy consumption due to exponentially increase in path-loss with the distance between source and sink of information. In multihop networks, there are several sections (also can be called clusters) between the source and the sink nodes. Thus, the multihop sections closer to the sink should not only transmit their own information but also relay the information of other sections. So, the closer the section to the sink is, the higher energy it consumes and hence the sooner it dies [5]. Therefore, minimizing the total energy consumption of these multihop networks results in dying of closer sections to the sink, which itself reduces the lifetime of the network. This motivated us to mathematically derive the optimum sectoring (section sizes) of a multihop cooperative WSN to maximize its lifetime.

\section{Network Model}

In a typical multihop cooperative WSN, the network will be triggered by an event whose information should be collected by a local V-MIMO unit and then be transmitted by that unit to a sink. If the sink is far from the event, the information should be forwarded to the sink by a multihop-based routing manner [3].

To facilitate the analysis, we consider a linear network topology for such cooperative WSNs. The network with a node density $\beta$ (nodes $/ \mathrm{m}$ ) can be divided into $m$ sections (the same clusters for a general configuration) each with one information collecting/forwarding V-MIMO unit containing $N_{T}$ sensor nodes. The network length is $D$ and a section length $d_{i}$ (distance between $i$-th section 


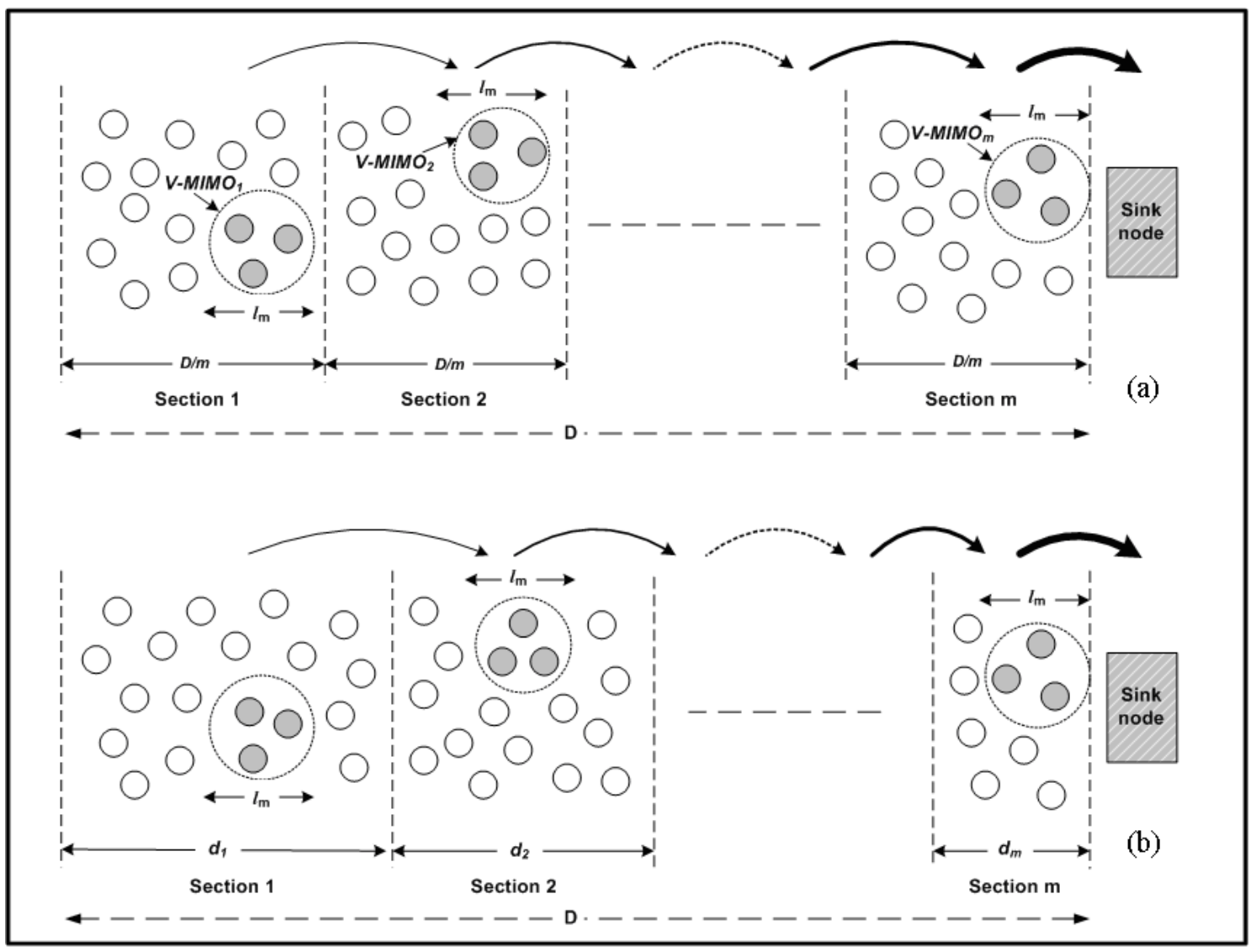

Figure 1. Network sectoring. (a) uniform sectoring; (b) optimal sectoring.

boundaries) is considered for each section (as depicted in Figure 1(a)).

Total energy consumption for transmitting $L$ bits in a MIMO system over a Rayleigh fading channel can be expressed as [3]

$$
E=L(1+\alpha) \bar{E}_{b} G_{0}(d)^{k}+L\left(P_{c t}+P_{c r}\right) / R_{b}
$$

where $\alpha$ is a parameter describing the efficiency of radio frequency power amplifier. $\bar{E}_{b}$ is the average energy per bit at the receiver for a given bit error rate requirement. $G_{0}$ is the power attenuation factor at unit distance $(d=1 \mathrm{~m})$ and $k$ is the path-loss exponent. $P_{c t}$ and $P_{c r}$ are the circuitry power consumption at the transmitter and receiver sides, respectively. $R_{b}$ is the bit rate [3-5]. Simply, if the sensor nodes are uniformly distributed in the network, the average number of bits generated in each section will be equal $L_{1}=L_{2} \cdots=L_{m}=L$. The scenario for information transmission to the sink in this network can be explained in the following three phases.

Phase 1: In each section, the local information is collected by a V-MIMO unit (a group of $N_{T}$ nodes). Consider that generalization for multiple V-MIMO units in each section is straight forward.

Phase 2 (non-cooperative communication): In each section, the $N_{T}$ nodes broadcast their information to all the other V-MIMO nodes using different time slots with uncoded $M$-QAM [3]. Meanwhile, these $N_{T}$ nodes are placed beside each other with a maximum separation of $l_{m}=N_{T} / \beta$ meters. For these transmissions, we consider a Rayleigh fading channel with square-law path-loss plus additive white Gaussian noise (AWGN). Energy consumption of the $i$-th section for the non-cooperative phase can be expressed as [5]

$$
E_{\text {intra }}^{i}=L(1+\alpha) \bar{E}_{b 2} G_{0}\left(l_{m}\right)^{2}+L\left(P_{c t}+P_{c r}\right) /\left(b_{2} B W\right)
$$

where $b_{2}$ represents the modulation constellation size, $\bar{E}_{b 2}$ denotes $\bar{E}_{b}$ for constellation size $b_{2}$ and $B W$ is the transmission bandwidth of each sensor node.

Phase 3 (cooperative communication): When the total information is collected by a V-MIMO unit, it will be encoded and transmitted using practical orthogonal STBC code design explained in [6] to the V-MIMO unit of the next section. This code design allows us to obtain the energy consumption with best accuracy. It is notable that each of these V-MIMO units should relay the information of previous units as well. For these transmissions, we consider a Rayleigh fading channel with $k$-th order 
path-loss plus AWGN.

In order to take the effect of training overheads for channel estimation into account, suppose that the information block size of the STBC code is equal to $F$ symbols and in each block we include $N$ training symbols. Hence, if $R$ is the transmission rate [4,5], the effective bit rate of the MIMO transmission is given by

$$
R_{e f f}=\frac{F-N}{F} \times R \times\left(b_{1} B W\right)
$$

where $b_{1}$ represents the modulation constellation size for the cooperative communications. Hence, by considering (1) and (3), energy consumption of each section for multihop transmissions (cooperative communications) can be given by

$$
E_{\text {inter }}^{i}=i L(1+\alpha) \bar{E}_{b 1} G_{0}\left(d_{i}\right)^{k} \times \frac{b_{1} B W}{R_{e f f}}+\frac{i L\left(N_{T} P_{c t}\right)}{R_{e f f}}+\frac{(i-1) L\left(N_{T} P_{c t}\right)}{R_{e f f}}
$$

where $\bar{E}_{b 1}$ denotes $\bar{E}_{b}$ for constellation size $b_{l}$. In the above equation, the first term denotes the energy consumption for transmitting $i L$ bits ( $L$ bits collected by the $i$-th V-MIMO plus $(i-1) L$ bits received from $(i-1)$ previous sections), the second and the third terms denote the circuitry energy consumption for transmitting $i L$ and receiving $(i-1) L$ bits. Therefore, the total energy consumption of each section by considering both cooperative and non-cooperative communications can be expressed as $E_{i}=E_{\text {intra }}^{i}+E_{\text {inter }}^{i}$.

\section{Optimal Network Sectoring}

As mentioned earlier, maximizing the lifetime of the network is our main goal. In the explained cooperative network model, the lifetime of the network is directly dependent upon the existence of each section. It means that if one section dies because of its high workload, the network connectivity will be broken and the network will become useless. Hence, the effective lifetime of the network is inversely proportional to the maximum energy consumption (workload) among the network sections as

$$
\text { Lifetime } \propto \frac{1}{\max \left(E_{i}\right)}, \quad i=1, \cdots, m .
$$

Consider Figure 1(a) where the network has a uniform sectoring. In this case, the energy consumption for the non-cooperative communication (as in (2)) is approximately equal for all sections. Therefore, knowing the fact that each section should not only transmit its own information but also forward the information of previous sections, the workload of the sections closer to the sink is much higher. Thus, with equal-size sections, the network lifetime is limited to the lifetime of the $m$-th section (the last section), whose lifetime is much shorter than other sections. Obviously, this is not the optimal performance for this network.

In order to maximize the network lifetime, we propose to modify the section sizes as illustrated in Figure 1(b). To find the optimal network sectoring, the following optimization problem should be solved for the linear network under consideration

$$
\begin{aligned}
& \min \max \left(E_{i}\right), \\
& \text { s.t } \sum_{i=1}^{m} d_{i}=D .
\end{aligned}
$$

Lemma: Instead of solving the above optimization problem, we solve the following equivalent optimization problem problem

$$
\begin{aligned}
& \min \operatorname{Var}\left(E_{i}\right)=1 / m \sum_{i=1}^{m} E_{i}^{2}-\left(1 / m \sum_{i=1}^{m} E_{i}\right)^{2}, \\
& \text { s.t } \sum_{i=1}^{m} d_{i}=D .
\end{aligned}
$$

For the simplicity, by considering (2) and (4), we rewrite $E_{i}$ as $E_{i}=i A d_{i}^{k}+i B+C$, where

$$
\begin{aligned}
& A=L(1+\alpha) \bar{E}_{b 1} G_{0} \frac{b_{1} B W}{R_{e f f}}, \\
& B=\frac{L\left(N_{T} P_{c t}\right)}{R_{e f f}}+\frac{L\left(N_{T} P_{c r}\right)}{R_{e f f}}, C=-\frac{L\left(N_{T} P_{c r}\right)}{R_{e f f}} .
\end{aligned}
$$

Hence, we will have

$$
\begin{aligned}
& \min 1 / m \sum_{i=1}^{m}\left(i A d_{i}^{k}+i B+C\right)^{2}-\left(1 / m \sum_{i=1}^{m}\left(i A d_{i}^{k}+i B+C\right)\right)^{2}, \\
& \text { s.t } \sum_{i=1}^{m} d_{i}=D .
\end{aligned}
$$

To solve the above problem, we define the Lagrangian $(J)$ [7] associated with the problem (9) as

$$
J=\operatorname{Var}\left(E_{i}\right)+\lambda\left(D-\sum_{i=1}^{m} d_{i}\right) .
$$

By setting the first derivative of $J$ with respect to each of its variables equal to zero, we have

$$
\begin{gathered}
\frac{\partial J}{\partial d_{j}}=\frac{2 E_{j}}{m} \frac{\partial E_{j}}{\partial d_{j}}-\frac{2 \sum_{i=1}^{m} E_{i}}{m^{2}} \frac{\partial E_{j}}{\partial d_{j}}-\lambda=0, \quad j=1, \cdots, m, \\
\frac{2 E_{j}}{m}-\frac{2 \sum_{i=1}^{m} E_{i}}{m^{2}}=\lambda\left(\frac{\partial E_{j}}{\partial d_{j}}\right)^{-1} .
\end{gathered}
$$

To extract $E_{j}$, we take a summation over $j$ from both sides of (12), which results in

$$
\begin{gathered}
\frac{2}{m} \sum_{j=1}^{m} E_{j}-\frac{2}{m} \sum_{i=1}^{m} E_{i}=\lambda \sum_{j=1}^{m}\left(\frac{\partial E_{j}}{\partial d_{j}}\right)^{-1}, \\
0=\lambda \sum_{j=1}^{m}\left(\frac{\partial E_{j}}{\partial d_{j}}\right)^{-1} .
\end{gathered}
$$


From (2) and (4), ( $E_{j}$ is strictly ascending function of $d_{j}$ ) and hence $\lambda=0$. By substituting $\lambda=0$ into (12), we have

$$
E_{1}=E_{2}=\cdots=E_{m}=\frac{\sum_{i=1}^{m} E_{i}}{m}=E .
$$

As is expected, Equation (15) means that to maximize the lifetime of the network, all of the sections should have equal workload and therefore all die together. From summation of (2) and (4) and considering (15), the optimal section size can be expressed as

$$
d_{i}=\sqrt[k]{\frac{E-C-i B}{i A}} \quad i=1, \cdots, m .
$$

As is clear form (16), the optimal network sectoring problem has a waterfilling form solution $\left(d_{i}\right)$ with parameter $E$ given by (15), which is determined by network length constraint $D$.

Proof of the Lemma: We prove that (6) and (7) have the same solutions. Assume that these problems have different solutions $\left(d_{i}^{6}, E_{i}^{6}\right)$ and $\left(d_{i}^{7}, E_{i}^{7}\right)$ for $\forall i$ respectively. Note that $E_{i}^{7}$ values are equal $\forall i$ as discussed in (15). Since the network length constraint should hold for both solutions, we cannot say $d_{i}^{6} \leq d_{i}^{7} \forall i$. So, there should be an $i$ so that $d_{i}^{6}>d_{i}^{7}$ (unless $d_{i}^{6}=d_{i}^{7}$ ) and $E_{i}^{6}>E_{i}^{7}$. This means that $\left(d_{i}^{7}, E_{i}^{7}\right)$ is a better solution for problem (6); in other words, $\left(d_{i}^{6}, E_{i}^{6}\right)$ is not the optimal solution for (6). Hence, our initial assumption was wrong and therefore (6) and (7) have the same solution.

\section{Performance Evaluation}

We consider the explained network model in Section 2 and evaluate the effect of the proposed network sectoring for a linear $m$-section WSN using computer simulations. We consider the case where $m=8, N_{T}=4, b_{1}=2$ and $b_{2}$ $=8$. The path-loss exponent is considered to be $k=2$ for non-cooperative transmissions and $k=3$ for cooperative transmissions. As described earlier, the lifetime of the network is inversely proportional to maximum energy consumption of individual sections as in (5). Thus, to represent the results, we define $1 / \max \left(E_{i}\right), i=1,2, \cdots, m$ as lifetime coefficient.

Figure 2 compares the total energy consumption of different sections of the multihop network for the case of uniform and the proposed optimal sectoring for $D=1000 \mathrm{~m}$. The $x$-axis represents the boundaries of different sections from the first section that is located at $x=0$. From the figure, the proposed optimal sectoring (section sizes) results in equal total energy consumption for different sections of the network, which in turn leads to maximizing of the total network lifetime. Meanwhile, in the uniform sectoring the closest section to the sink consumes

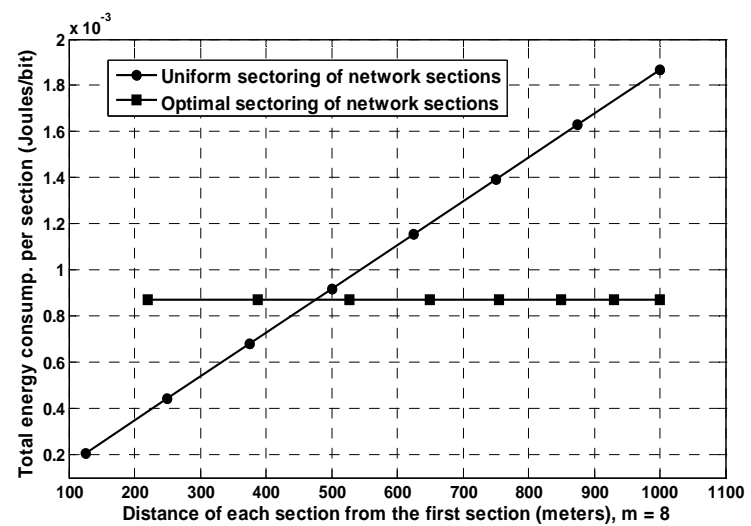

Figure 2. Total energy consumption of different sections.

much more energy compared to that of the optimal sectoring, and hence the uniformly sectored network will die sooner. As is expected from our previous analysis, to achieve even energy distribution in the explained network, the sections which are closer to the sink have smaller sizes.

Figure 3 illustrates the lifetime coefficient of uniform and the proposed optimal sectoring. As well, relative lifetime ( $R=$ Lifetime coeff. of optimal/Lifetime coeff. of uniform) for both schemes is depicted for different network sizes $(D)$. From the figure, the proposed optimal sectoring enhances the lifetime of the network by about $130 \%$ (2.3 times) for large network sizes $(D)$ compared to that of the uniform sectoring.

\section{Conclusions}

In this paper, we investigated the effect of section (cluster) size on the total power consumption of multihop cooperative WSNs. We proposed an optimal network sectoring for linear multihop networks so that it minimizes the total power consumption of the network by evenly distributing the power in such a network. The simulation results illustrate that the proposed optimal

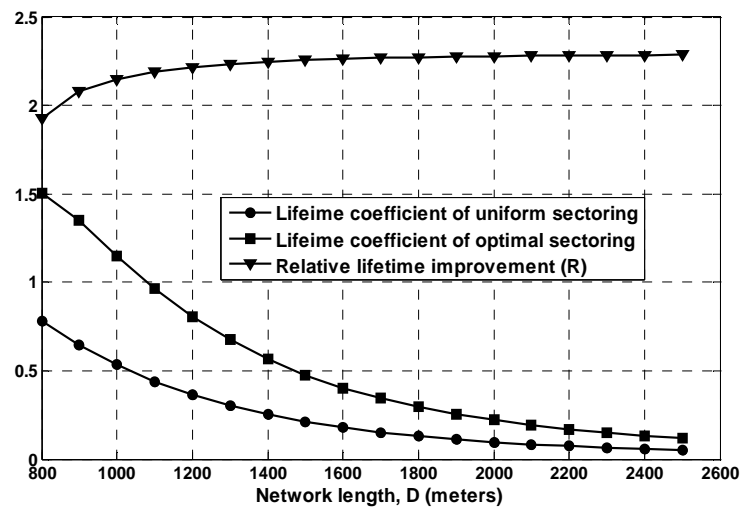

Figure 3. Lifetime of network versus network size, $D$. 
sectoring scheme leads to equal power consumption in all the sections and increases the lifetime of the network up to 2.3 times compared to the equal sectoring.

\section{Acknowledgements}

The authors would like to acknowledge Mr. Mehran Mashreghi for his insightful comments and helpful discussions.

\section{References}

[1] S. Alamouti, "A Simple Transmit Diversity Technique for Wireless Communications,” IEEE Journal on Selected Areas in Communications, Vol. 16, No. 8, October 1998, pp. 1451-1458.

[2] J. N. Laneman, D. N. C. Tse and G. W. Wornell, "Cooperative Diversity in Wireless Networks: Effcient Protocols and Outage Behaviour," IEEE Transactions on Informormation Theory, Vol. 50, No. 12, December 2004, pp. 3062-3080.
[3] S. Cui, A. J. Goldsmith and A. Bahai, "Energy-Efficiency of MIMO and Cooperative MIMO Techniques in Sensor Networks," IEEE Journal on Selected Areas in Communications, Vol. 22, No. 6, August 2004, pp. 1089-1098.

[4] S. K. Jayaweera, "Virtual MIMO-Based Cooperative Communication for Energy-Constrained Wireless Sensor Networks," IEEE Transactions on Wireless Communications, Vol. 5, No. 5, May 2006, pp. 984-989.

[5] M. Mashreghi and B. Abolhassani, "Prolongation of Lifetime for Wireless Sensor Networks," Proceedings of International Conference on Intelligent Sensors, Sensor Networks and Information Processing, Melbourne, 3-6 December 2007, pp. 73-78.

[6] K. Lu, S. Fu and X.-G. Xia, "Closed Form Designs of Complex Orthogonal Space-Time Block Codes of Rates $(\mathrm{k}+1) /(2 \mathrm{k})$ for $2 \mathrm{k}_{1}$ or $2 \mathrm{k}$ Transmit Antennas," Proceedings of IEEE International Symposium on Information Theory, Chicago, 27 June-2 July 2004, p. 307.

[7] H. S. Boyd and L. Vandenberghe, "Convex Optimization," Cambridge University Press, Cambridge, 2004. 